\title{
ON SHOCKS DRIVEN BY HIGH-MASS PLANETS IN RADIATIVELY INEFFICIENT DISKS. II. THREE- DIMENSIONAL GLOBAL DISK SIMULATIONS
}

\author{
Wladimir Lyra ${ }^{1,2,3}$, Alexander J. W. Richert ${ }^{4}$, Aaron Boley ${ }^{5}$, Neal Turner ${ }^{2}$, Mordecai-Mark Mac Low ${ }^{6,7}$, \\ Satoshi OKUZumi ${ }^{2,8}$, and Mario Flock ${ }^{2}$ \\ ${ }^{1}$ Department of Physics and Astronomy, California State University Northridge, 18111 Nordhoff St, Northridge, CA 91330, USA; wlyra@csun.edu \\ 2 Jet Propulsion Laboratory, California Institute of Technology, 4800 Oak Grove Drive, Pasadena, CA 91109, USA; \\ neal.j.turner@jpl.nasa.gov, mario.flock@jpl.nasa.gov \\ ${ }^{3}$ Division of Geological \& Planetary Sciences, California Institute of Technology, 1200 E California Blvd MC 150-21, Pasadena, CA 91125, USA \\ ${ }^{4}$ Department of Astronomy \& Astrophysics, Penn State University, 525 Davey Lab, University Park, PA 16802, USA; ajr327@psu.edu \\ 5 Department of Physics and Astronomy, University of British Columbia, 6224 Agricultural Road, Vancouver, BC V6T 1Z1, Canada; acboley@phas.ubc.ca

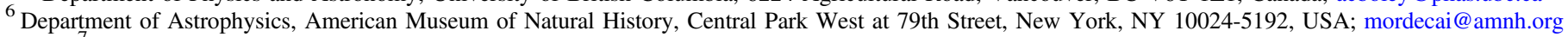 \\ ${ }^{7}$ Institut für Theoretische Astrophysik, Zentrum für Astronomie der Universität Heidelberg, Albert-Ueberle-Str. 2, D-69121 Heidelberg, Germany

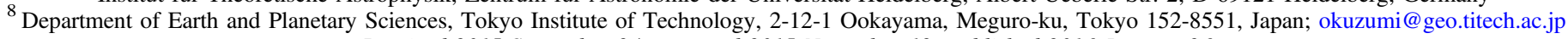 \\ Received 2015 September 24; accepted 2015 November 12; published 2016 January 26
}

\begin{abstract}
Recent high-resolution, near-infrared images of protoplanetary disks have shown that these disks often present spiral features. Spiral arms are among the structures predicted by models of disk-planet interaction and thus it is tempting to suspect that planetary perturbers are responsible for these signatures. However, such interpretation is not free of problems. The observed spirals have large pitch angles, and in at least one case (HD 100546) it appears effectively unpolarized, implying thermal emission of the order of $1000 \mathrm{~K}$ ( $465 \pm 40 \mathrm{~K}$ at closer inspection). We have recently shown in two-dimensional models that shock dissipation in the supersonic wake of high-mass planets can lead to significant heating if the disk is sufficiently adiabatic. Here we extend this analysis to three dimensions in thermodynamically evolving disks. We use the Pencil Code in spherical coordinates for our models, with a prescription for thermal cooling based on the optical depth of the local vertical gas column. We use a $5 M_{J}$ planet, and show that shocks in the region around the planet where the Lindblad resonances occur heat the gas to substantially higher temperatures than the ambient gas. The gas is accelerated vertically away from the midplane to form shock bores, and the gas falling back toward the midplane breaks up into a turbulent surf. This turbulence, although localized, has high $\alpha$ values, reaching 0.05 in the inner Lindblad resonance, and 0.1 in the outer one. We find evidence that the disk regions heated up by the shocks become superadiabatic, generating convection far from the planet's orbit.
\end{abstract}

Key words: hydrodynamics - planet-disk interactions - planets and satellites: formation - protoplanetary disks shock waves - turbulence

\section{INTRODUCTION}

Recent observations of protoplanetary disks reveal clear spiral features (e.g., Muto et al. 2012; Garufi et al. 2013; Currie et al. 2014; Benisty et al. 2015), as seen in polarized scattered light in near-infrared. Given the high density of the disk, the micron-sized dust grains responsible for the scattering must be suspended high in the disk atmosphere to produce these features.

Numerical simulations of planet-disk interaction (e.g., Bryden et al. 1999; Kley 1999; Lubow et al. 1999; Baruteau et al. 2014) have shown the formation of distinctive one-armed spirals. Even the very first of such calculations (Miki 1982), although done in two dimensions (2D) in a local box, already shows evidence of a spiral pattern excited by the planet. Highmass planets can lead to strong spiral shocks, while low-mass planets (e.g., Neptune) produce spirals with only slight density enhancements. In the low-mass limit, the perturbations can be treated using linear theory, and Ogilvie \& Lubow (2002) showed that the shape of the spiral density perturbation launched by such a low-mass planet can be predicted analytically as a superposition of the individual Fourier modes.

Although it is tempting to assume that the observed spiral features are indeed due to planets, several of the observed spirals have pitch angles too wide to fit the analytical predictions based on linear theory in the simplest case of an azimuthally isothermal disk. Since the pitch angle in the linear theory depends on the sound speed, this implies that the spirals are launched from higher temperatures than the ambient gas. MWC 758, for instance has a measured background temperature of $\approx 53 \mathrm{~K}$ at $50 \mathrm{AU}$ (Isella et al. 2010), yet a fit to the analytical model requires a temperature of $\approx 300 \mathrm{~K}$ to match the observed spirals (Benisty et al. 2015).

Nonetheless, if planets do produce these spirals, the simplest explanation for the observed spiral morphologies may be that the planets are too massive for their influence on the disk to be described by linear theory. The linear theory requires the planets to be of low mass, so that the wake launched is subsonic. If the planet is massive, the wake launched propagates faster than the speed of sound, and steepens into a shock near the planet. The shape of the spiral shock then rapidly deviates from that predicted from linear theory.

Evidence of this behavior has been abundantly clear in the literature for at least a decade, visibly seen in the disk-planet simulations in the code comparison of de Val-Borro et al. (2006). In that study, 14 independent codes were used to reproduce viscous and inviscid simulations of Neptune-mass and Jupiter-mass planets. Whereas the shape of the spirals launched by the Neptune-mass planets were described well by linear theory (Ogilvie \& Lubow 2002), the spirals launched by the Jupiter-mass planets deviated from it substantially. It was 
understood that this feature was due to the Jupiter-mass planet driving shocks that were outside the range of applicability of linear theory. Dong et al. (2015) and Zhu et al. (2015) have recently shown that a weakly nonlinear theory (Goodman \& Rafikov 2001; Rafikov 2002) can reproduce the pitch angle of spiral shocks launched by high-mass planets.

Yet, even though a higher pitch angle can reproduce the spiral launched by high-mass planets reasonably well, without requiring ad hoc high aspect ratios, there are still features that require high temperatures. For instance, in the circumstellar material around HD 100546, a spiral-like feature is seen (Currie et al. 2014, 2015) showing little polarization. This implies that the emission is thermal rather than scattered starlight, and thus must of the order of $1000 \mathrm{~K}$ to reproduce the infrared brightness (in Section 4 we examine the observational data and determine the temperature needed to reproduce the $H$ and $L^{\prime}$ magnitudes to be $465 \pm 40 \mathrm{~K}$ ).

We have recently shown in Richert et al. (2015, hereafter Paper I) that when a planet is massive enough to produce a spiral wave that steepens into a shock, the associated shock dissipation can significantly heat the disk. If the radiative efficiency of the disk is low, the temperature can rise until buoyancy upsets the force balance. This can cause disk turbulence with significant angular momentum transport. Effectively, the gravitational potential well of the planet powers a vigorous heat source.

Here we report three-dimensional (3D) simulations exploring the same behavior. There are significant qualitative differences between $2 \mathrm{D}$ and $3 \mathrm{D}$ in this problem. The midplane is close to adiabatic, but the atmosphere of the disk behaves more isothermally because it can more quickly cool radiatively. This effect alone will already lead to a different behavior in 3D, since now the energy buildup due to shocks has to occur within the time that it takes for energy to diffuse from the midplane to the atmosphere (from which it escapes efficiently). Also, upward motions that bring a gas parcel from the midplane into the atmosphere will quickly lead to efficient cooling. Another major difference is that a shock will be weaker in $3 \mathrm{D}$, because the extra dimension translates into an extra degree of freedom. Therefore, when the shock expands adiabatically, it can accelerate material in the vertical direction. This was discussed in detail in Boley \& Durisen (2006), who referred to the structure as shock bores, following the work of Gomez \& Cox (2004) on galactic disk shocks. As gas shocks and accelerates upwards, a column of hot, thin gas forms around the colder and denser gas just outside of the spiral shocks. When the jetted material falls back toward the disk surface, breaking waves can form, similar in appearance to beach surf. If strong enough, these waves should produce localized turbulence around the locations of the Lindblad resonance. We intend in this paper to find signatures of this process in 3D, non-isothermal disks containing high-mass planets.

Although our work resembles that of Zhu et al. (2015) in that we both model 3D, nonisothermal disks with high-mass planets in order to examine the effect of their shocks, there are also significant differences in technique and focus. While Zhu et al. (2015) use a fixed cooling time with an exponential fall to isothermal beyond three scale heights, we use a dynamical cooling time dependent on vertical optical depth, that varies following the movements of the material. Zhu et al. (2015) examined the shape of the spiral launched by the planet, while we focus on the effect of the shocks on disk accretion and energy budget. Because of that, Zhu et al. only ran their disks for 10 orbits, halting before gap opening and substantial alteration of the disk structure, while we ran to 40 orbits, long enough to achieve gap opening.

This paper is structured as follows. In Section 2 we describe the model used. The results are presented in Section 3, followed by discussions and conclusions in Section 4.

\section{THE MODEL}

\subsection{Model Equations}

The models shown in this paper were calculated with the Pencil Code. ${ }^{9}$ We solve the equations of hydrodynamics in spherical coordinates in the reference frame with the origin at the star and following the motion of the planet. In this frame, both the star and the planet are stationary. The equations of motion are

$$
\begin{gathered}
\frac{D \ln \rho}{D t}=-\nabla \cdot \boldsymbol{u}, \\
\frac{D \boldsymbol{u}}{D t}=-\frac{1}{\rho} \nabla p-\nabla \Phi+\frac{1}{\rho} \boldsymbol{\nabla} \cdot \boldsymbol{\zeta} \\
-2 \boldsymbol{\Omega}_{p} \times \boldsymbol{u}-\boldsymbol{\Omega}_{p} \times\left(\boldsymbol{\Omega}_{p} \times \boldsymbol{r}\right), \\
T \frac{D s}{D t}=-c_{V} \frac{\left(T-T_{\mathrm{ref}}\right)}{t_{\text {cool }}}+\Gamma_{\mathrm{sh}} .
\end{gathered}
$$

In these equations, $\rho$ is the density, $t$ the time, $\boldsymbol{u}$ the velocity, $p$ the pressure, $\Phi$ the gravitational potential, $\zeta$ the shock viscosity tensor, $\boldsymbol{\Omega}_{p}$ the angular velocity vector of the planet, $\boldsymbol{r}$ the distance to the origin, $s$ the entropy, $T$ temperature, $c_{V}$ the specific heat at constant volume, $T_{\text {ref }}$ a reference temperature, $t_{\text {cool }}$ the cooling time, and $\Gamma_{\text {sh }}$ the shock heating. The advective derivative

$$
\frac{D}{D t} \equiv \frac{\partial}{\partial t}+\boldsymbol{u} \cdot \nabla
$$

The gravitational potential

$$
\Phi=-\frac{G M_{\star}}{r}-\frac{G M_{p}}{\left(\left|\boldsymbol{r}-\boldsymbol{r}_{p}\right|^{2}+b^{2}\right)^{1 / 2}}+\frac{G M_{p}}{r_{p}^{3}} \boldsymbol{r} \cdot \boldsymbol{r}_{p}
$$

includes the contribution of the star (first term), planet (second term), and the indirect terms due to the motion of the star (third term). Here, $G$ is the gravitational constant, $M_{\star}$ the star mass, $M_{p}$ the planet mass, and $\boldsymbol{r}_{p}=r_{p} \hat{\boldsymbol{x}}$ the vector position of the planet. The softening radius $b$ in the planet potential is included to avoid singularities, and is set to the planet's Hill radius. Note that the planet's orbital frequency

$$
\boldsymbol{\Omega}_{p}=\left(\frac{G\left(M_{\star}+M_{p}\right)}{r_{p}^{3}}\right)^{1 / 2} \hat{z} .
$$

In Pencil, we capture shocks using an explicit shock viscosity prescription as first proposed by von Neumann \& Richtmyer (1950). The third term in the momentum equation (Equation (2)) is the viscosity required to spread shocks out to resolvable width, and the last term in the entropy equation (Equation (3)) is the shock viscous heating.

\footnotetext{
9 The code, with improvements done for this work, is publicly available at http://pencil-code.nordita.org.
} 
The shock viscosity tensor takes the form of a bulk viscosity

$$
\boldsymbol{\zeta}_{i j}=\nu_{\mathrm{sh}} \rho \delta_{i j} \nabla \cdot \boldsymbol{u},
$$

and the associated shock heating is

$$
\Gamma_{\mathrm{sh}}=\nu_{\mathrm{sh}}(\boldsymbol{\nabla} \cdot \boldsymbol{u})^{2} \text {. }
$$

They depend on the shock viscosity, which we define numerically as

$$
\nu_{\mathrm{sh}}=c_{\mathrm{sh}}\left\langle\max _{3}\left[(-\boldsymbol{\nabla} \cdot \boldsymbol{u})^{+}\right]\right\rangle[\min (\Delta x)]^{2} .
$$

The actual form of the shock viscosity that we use has been described in Haugen et al. (2004) and in Paper I. The superscript plus sign indicates the positive part of the quantity. In Paper I we experimented with a range of values for the coefficient $c_{\mathrm{sh}}$, and found that it did not affect the outcome of the simulations. As long as the shock is resolved, the value of the shock viscosity coefficient does not change the amount of heating; rather, it just changes the volume (number of grid cells) over which the shock energy is spread.

We use for equation of state

$$
p=\rho c_{\mathrm{s}}^{2} / \gamma
$$

where $c_{s}$ is the adiabatic sound speed, related to the temperature by

$$
T=c_{\mathrm{s}}^{2} /\left[c_{p}(\gamma-1)\right]
$$

$c_{p}=\gamma c_{V}$ is the specific heat at constant pressure, and $\gamma$ the adiabatic index. The specific heats are related to the universal gas constant $\mathcal{R}$ by

$$
\mathcal{R}=\mu\left(c_{p}-c_{V}\right),
$$

where $\mu$ is the mean molecular weight of the gas.

In the entropy equation, we use Newton cooling with a dynamical cooling time $t_{\text {cool }}$ that varies following the movements of the material. We define $t_{\text {cool }}$ based on the radiative timescale $t_{\mathrm{rad}}=E / \dot{E}$, where $E=c_{V} \rho T$ is the gas internal energy. Equating $\dot{E}=\boldsymbol{\nabla} \cdot \boldsymbol{F}$ and integrating to remove the divergence, we define

$$
t_{\mathrm{cool}} \equiv \frac{\int E d V}{\int F \hat{\boldsymbol{n}} \cdot d \boldsymbol{A}} .
$$

Here, the flux $F=|\boldsymbol{F}|=\sigma T^{4} / \tau_{\text {eff }}$ and the effective optical depth, accounting for both the opaque and transparent limits, is given by (Hubeny 1990, D’Angelo et al. 2003)

$$
\tau_{\text {eff }}=\frac{3 \tau}{8}+\frac{\sqrt{3}}{4}+\frac{1}{4 \tau} .
$$

For the integration we assume a Gaussian sphere of radius equal to the scale height, the largest isotropic scale

$$
H=\frac{1}{\sqrt{\gamma}} \frac{c_{s}}{\Omega_{K}},
$$

where

$$
\Omega_{K}^{2}=\frac{G\left(M_{\star}+M_{p}\right)}{R^{3}} .
$$

is the Keplerian frequency. We assume as an approximation that $E$ and $F$ are constant in that sphere and equal to the value at the center, and thus write the cooling time as

$$
t_{\mathrm{cool}}=\frac{c_{V} \rho H \tau_{\mathrm{eff}}}{3 \sigma T^{3}} .
$$

This cooling prescription was used in Lyra et al. (2010) and Horn et al. (2012) for one-dimensional vertically averaged disk models. Averaging instead over cylinders or over horizontal layers yields different geometric factors (2 and 4/3, respectively, where we have 3; Horn et al. 2012, D'Angelo et al. 2003). The range among the geometrical factors is considerably less than the range in opacity allowed by dust abundance variations, so any of the three approaches could safely be used.

The dimensionless cooling time is defined as

$$
\beta \equiv t_{\text {cool }} \Omega_{K}
$$

The optical depth $\tau$ is the vertical integral of $\rho \kappa$ where $\kappa$ is the opacity, taken from Bell et al. (1997), updated by Semenov et al. (2004). For every grid cell, we calculate the optical depth due to material above and below

$$
\begin{gathered}
\tau_{\text {upper }}=-\int_{\infty}^{z} \rho \kappa d z^{\prime} \\
\tau_{\text {lower }}=\int_{-\infty}^{z} \rho \kappa d z^{\prime}
\end{gathered}
$$

and take $\tau=\min \left(\tau_{\text {upper }}, \tau_{\text {lower }}\right)$. In practice, instead of integrating in $z$ we simply integrate in $\theta$, which simplifies the amount of required communication in a spherical grid. Since the disk is geometrically thin, and the departure between surfaces of constant $z$ and surfaces of constant $\theta$ is more pronounced only for the optically thin, high- $z$ material, the error introduced is small. The reference temperature $T_{\text {ref }}$ is set to the initial temperature at every radius.

Sixth-order hyper-dissipation terms are added to the evolution equations to provide extra dissipation near the grid scale, as discussed in Lyra et al. (2008). These terms are needed for numerical stability because the high-order scheme of the Pencil Code has little overall numerical dissipation (McNally et al. 2012). They are chosen to produce Reynolds numbers of order unity at the grid scale, but then drop as the sixth power of the scale at larger scales, so that they have negligible influence on the large-scale flow.

\subsection{Initial Conditions}

We model the disk in spherical coordinates, using a logarithmic grid in the radial direction that samples a constant number of grid cells per scale height (in the radial direction). The grid is uniform in the meridional and azimuthal directions, and has resolution $\left(N_{r}, N_{\theta}, N_{\phi}\right)=(256,128,768)$. The radial range is $[0.4,2.5]$, the meridional range spans $4 H$ above and below the midplane, which corresponds to $[-0.28,0.28]$ radians $\left(\approx 16^{\circ}\right.$ to each side). The azimuthal range is $2 \pi$. The temperature is initially constant in cylinders

$$
T=T_{0}\left(\frac{R}{R_{0}}\right)^{q}
$$

where $R=r \sin \theta$ is the cylindrical radius. Solving the condition of hydrostatic equilibrium in the vertical direction, 
the density is

$$
\ln \rho=\ln \rho_{0}+p \ln \left(\frac{R}{R_{0}}\right)+(1-\sin \theta)\left(\frac{H}{R}\right)^{-2} .
$$

We use $\rho_{0}=1$ and $p=-1.5$, where $p$ is the slope of the radial scaling of the midplane density.

For these profiles of density and temperature, the condition of centrifugal equilibrium is satisfied for

$$
u_{\phi}=R\left(\xi \Omega_{K}-\Omega_{p}\right)
$$

where

$$
\xi^{2}=1+(p+q)\left(\frac{H}{R}\right)^{2}+q(1-\sin \theta) .
$$

We use units such that

$$
r_{0}=G\left(M_{\star}+M_{P}\right)=1
$$

and also $\rho_{0}=1$ as stated before. The other quantities are $c_{s 0}=0.07, \gamma=1.4, \mu=2.34, q=-1$, and $G M_{p}=5 \times 10^{-3}$ (that is, $G M_{\star}=0.995$ ). The inclusion of radiation physics breaks the scale-invariance of the code units. We use physical units such that the unit of length is the semimajor axis of Jupiter, 5.2 AU, $\left(7.785 \times 10^{13} \mathrm{~cm}\right)$, and the velocity unit is thus the circular velocity at Jupiter's orbit, $1.306 \times 10^{6} \mathrm{~cm} \mathrm{~s}^{-1}$. The unit of density is $2 \times 10^{-11} \mathrm{~g} \mathrm{~cm}^{-3}$, as in the minimum mass solar nebula (Hayashi 1981), and the unit of temperature is Kelvin.

The planetary mass is increased from zero to the full mass in ten orbits, to avoid the strong impact of introducing a highmass planet in a quiescent disk.

For boundary conditions in the radial direction, we use outflow for the radial velocity, symmetric (zero-gradient) for the meridional velocity, and constant gradient (zero second derivative) for the azimuthal velocity, density, and entropy. For the meridional direction, we use zero-gradient for the radial velocity, outflow for the meridional velocity, and constant gradient for azimuthal velocity and entropy. For the density we maintain the condition of vertical stratification (i.e., we extrapolate the Gaussian structure).

The optical depth calculated from this initial condition is plotted in the upper panel of Figure 1 while the dimensionless cooling time, $\beta=t_{\text {cool }} \Omega_{K}$, is shown in the lower panel. The expected behavior of nearly adiabatic in the midplane and nearly isothermal in the atmosphere is well reproduced.

\section{RESULTS}

We consider a model with a $5 M_{J}$ planet embedded in the disk model described in Section 2. In Figure 2 we show the shock structure in the disk, as traced by the shock viscosity Equation (9) after 40 orbits. (We normalize by $c_{\mathrm{s}} \mathrm{H}$ so that the shock viscosity is dimensionless.) The left panel shows the meridional plane that passes through the planet, while the right panel shows the midplane. Shocks are seen inward and outward from the planet. In the meridional plane they form shock columns that extend well into the disk atmosphere. In the upper atmosphere the shocks curve slightly toward the star, as also seen in Zhu et al. (2015), using a different method and cooling scheme. In the midplane the shocks follow spiral patterns. These shocks are the primary locations of energy dissipation, according to Equation (8). The actual temperature increase will depend on the local value of the cooling time $\beta$.
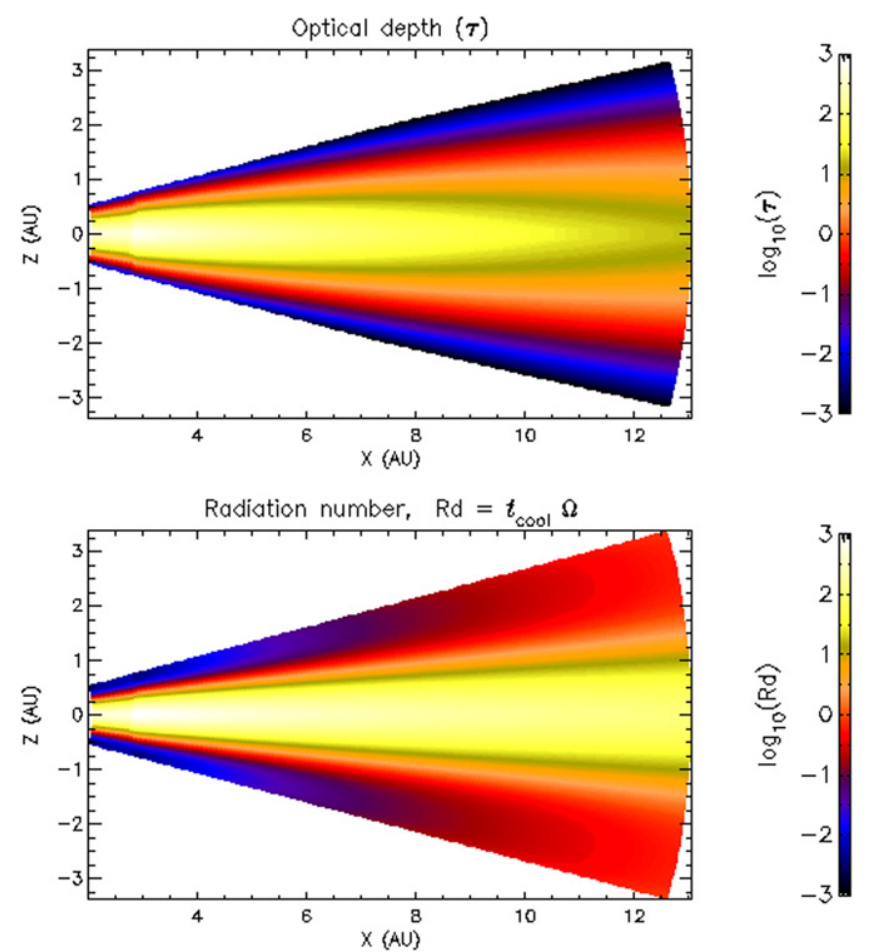

Figure 1. Optical depths and cooling times of our initial disk. The midplane is nearly adiabatic, while the upper layers are nearly isothermal. Calculated using the opacities of Bell et al. (1997), and Equation (17). The dimensionless cooling time is $\beta=t_{\text {cool }} \Omega_{K}$.

Figure 3 shows the temperature in the meridional plane. There are high-temperature lobes in the shock regions around the planet, and high temperatures are confined to the more adiabatic, high- $\beta$ region around the midplane. Above the height where $\beta \approx 1$ the temperature matches that of the quiescent gas. These hot lobes mark the positions of the shock bores. In this case, the lobes are at $450 \mathrm{~K}$, while the ambient gas is at $175 \mathrm{~K}$.

To understand the structure of the shocks we examine in Figure 4 the flow in the $\phi-\theta$ plane at the radial position of each lobe. The upper panel shows the temperature, and the lower panel the shock profile. The velocity field is superposed. The bore is seen extending almost all the way to the upper boundary. The flow upstream of the shock is quiescent, following Keplerian orbits. The downstream flow shows that material is accelerated upward, as expected from the analysis of Boley \& Durisen (2006). The upward moving gas reaches approximately $2 H$ before falling to the midplane again as it proceeds downstream.

A secondary weaker bow shock forms just downstream, leading to a smaller lobe of warm temperature. However, this bow shock does not seem to be associated with breaking waves: it is instead the result of the spiral feature formed by the lobe on the other side of the planet, as seen in Figure 2. The flow in the lobe to the right of the planet (right panels) shows the same structure, of upward acceleration in the vertical direction, fallback around $2 H$, and secondary bow shock.

In the upper panel of Figure 5 we plot the vertical velocity in the midplane, showing the result of the shocks. A turbulent structure is seen around the orbits at the radial location of the lobes that mark the shocks. In the lower panel of Figure 5 we plot the azimuthal velocity residual, $\delta u_{\phi}=u_{\phi}-\left\langle u_{\phi}\right\rangle$, in the midplane. The residual is supersonic near the planet, as expected, so we plot it only up to Mach numbers of 0.5 , to identify the turbulence. 

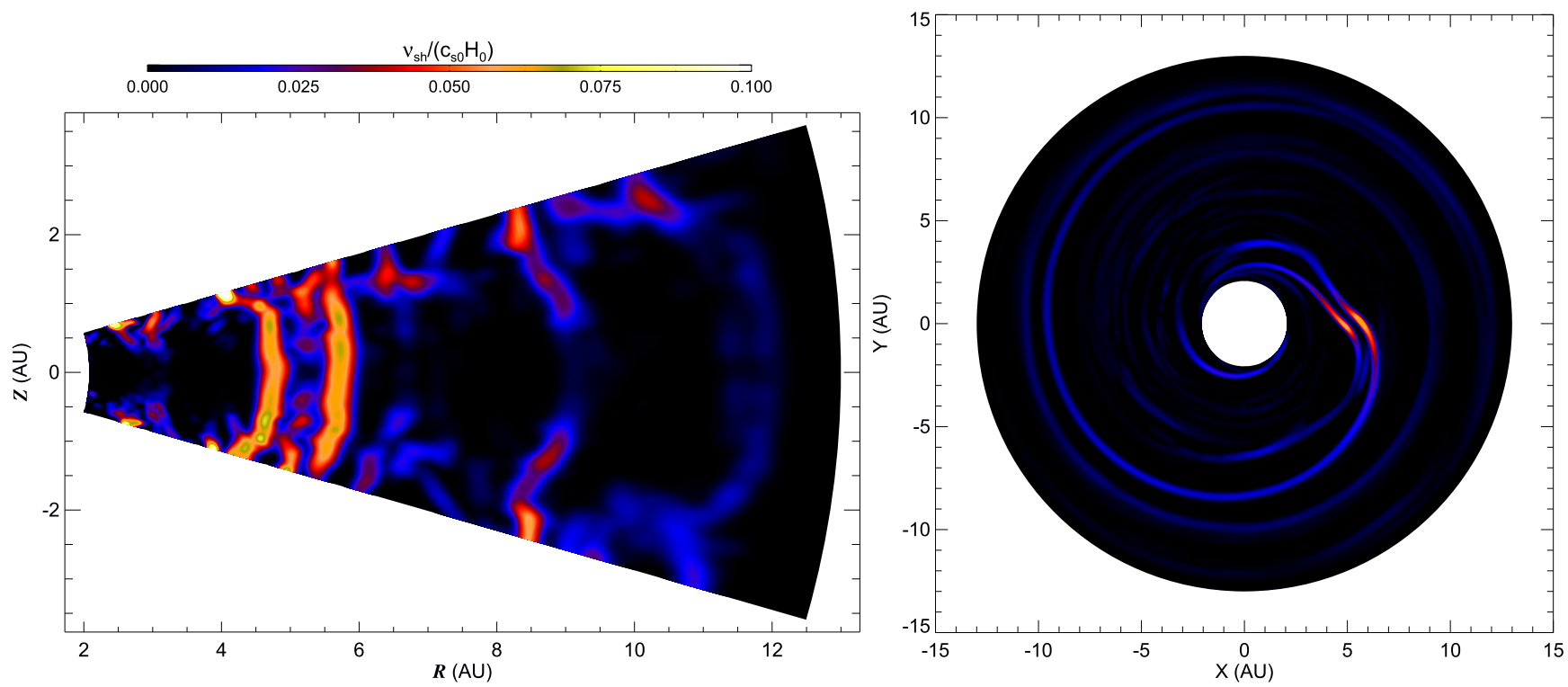

Figure 2. The location of shocks as traced by the shock viscosity Equation (9) normalized by $c_{\mathrm{s}} \mathrm{H}$, in the vertical (meridional) plane that contains the planet (left), and in the disk midplane (right). The shock viscosity is a measure of the positive part of $\boldsymbol{\nabla} \cdot \boldsymbol{u}$, so it only becomes significant in locations where shocks occur. The planet is located at 5.2 AU. Vertical shock bores are visible in the meridional plane, extending well into the disk atmosphere. In the midplane plot (right), the shocks are seen to be associated with the spiral the planet launches from its Lindblad resonances.

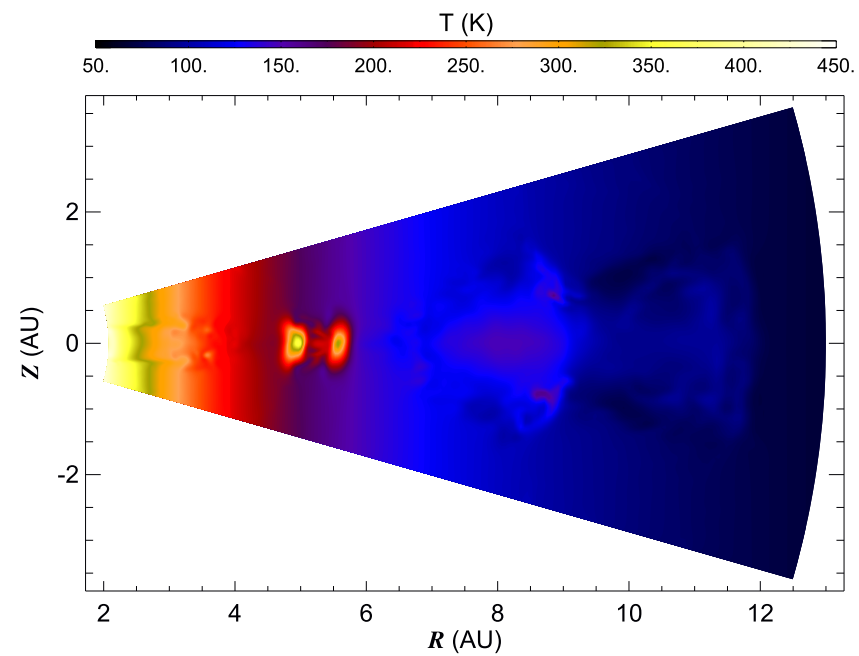

Figure 3. Temperature structure in the vertical plane containing the planet. Where the shock bore intersects the more radiatively inefficient regions of the disk, the temperature rises considerably.

We would like to quantify the level of angular momentum transport that is caused by this turbulence at the corotation radius of the region around $2 / 3 H$ where the Lindblad resonances cluster together (which we henceforth call the Lindblad lobe). For that we measure the alpha value (Shakura \& Sunyaev 1973),

$$
\begin{aligned}
& \alpha_{r \phi} \equiv \frac{\left\langle\rho \delta u_{r} \delta u_{\phi}\right\rangle}{\left\langle\rho c_{s}^{2}\right\rangle} \\
& =\frac{\left\langle\rho u_{r} u_{\phi}\right\rangle-\left\langle\rho u_{r}\right\rangle\left\langle u_{\phi}\right\rangle-\left\langle\rho u_{\phi}\right\rangle\left\langle u_{r}\right\rangle+\langle\rho\rangle\left\langle u_{r}\right\rangle\left\langle u_{\phi}\right\rangle}{\left\langle\rho c_{s}^{2}\right\rangle}
\end{aligned}
$$

This $\alpha_{r \phi}$ measures how much angular momentum is transported radially. We can also measure how much angular momentum is transported vertically (or meridionally) by computing

$$
\alpha_{\theta \phi} \equiv \frac{\left\langle\rho \delta u_{\theta} \delta u_{\phi}\right\rangle}{\left\langle\rho c_{s}^{2}\right\rangle} .
$$

We measure these quantities averaged in azimuth within $5^{\circ}$ above and below the midplane, which corresponds roughly to 1 scale height each way. We then average the snapshots from 10 to 40 orbits. The result is plotted in Figure 6. The upper panel shows the radial transport of angular momentum, the lower panel the vertical transport. We see that the vertical transport is essentially negligible, whereas the radial transport peaks in the region where the Lindblad resonances cluster together (which we henceforth call the Lindblad lobe) with an average of $\alpha \approx 0.05$ in the inner and $\alpha \approx 0.09$ in the outer. Frequently the $\alpha$ value in the outer Lindblad lobe exceeds 0.1 , characterizing strong accretion with a characteristic velocity $v_{\text {rms }} \equiv \sqrt{|\alpha|} c_{s} \approx 0.3 c_{s}$.

Some activity is also seen in the annulus around $10 \mathrm{AU}$. Comparing with Figure 3, we see this correlates with incipient rolls forming at $r=3.5,8.5$ and $11.5 \mathrm{AU}$. These appear to be buoyant expansion, radially and vertically, of the hot gas as it propagates outwards from the spiral shock. We tentatively identify the rolls as convection, which is also consistent with the indications of turbulent flow seen from 3 to $8 \mathrm{AU}$ in the midplane plot of Figure 2 (right panel) as well as Figure 5. To check that identification, we plot the entropy deviation (with respect to the initial condition) in the upper panel of Figure 7. We saturate the plot to avoid domination by the structures near the planet and highlight instead the variations in the outer disk. Indeed, a blob of high entropy is seen in the midplane, from about 8.5 to $11 \mathrm{AU}$.

To determine if this blob indeed would lead to convection, we determine if the entropy gradient is superadiabatic. The 

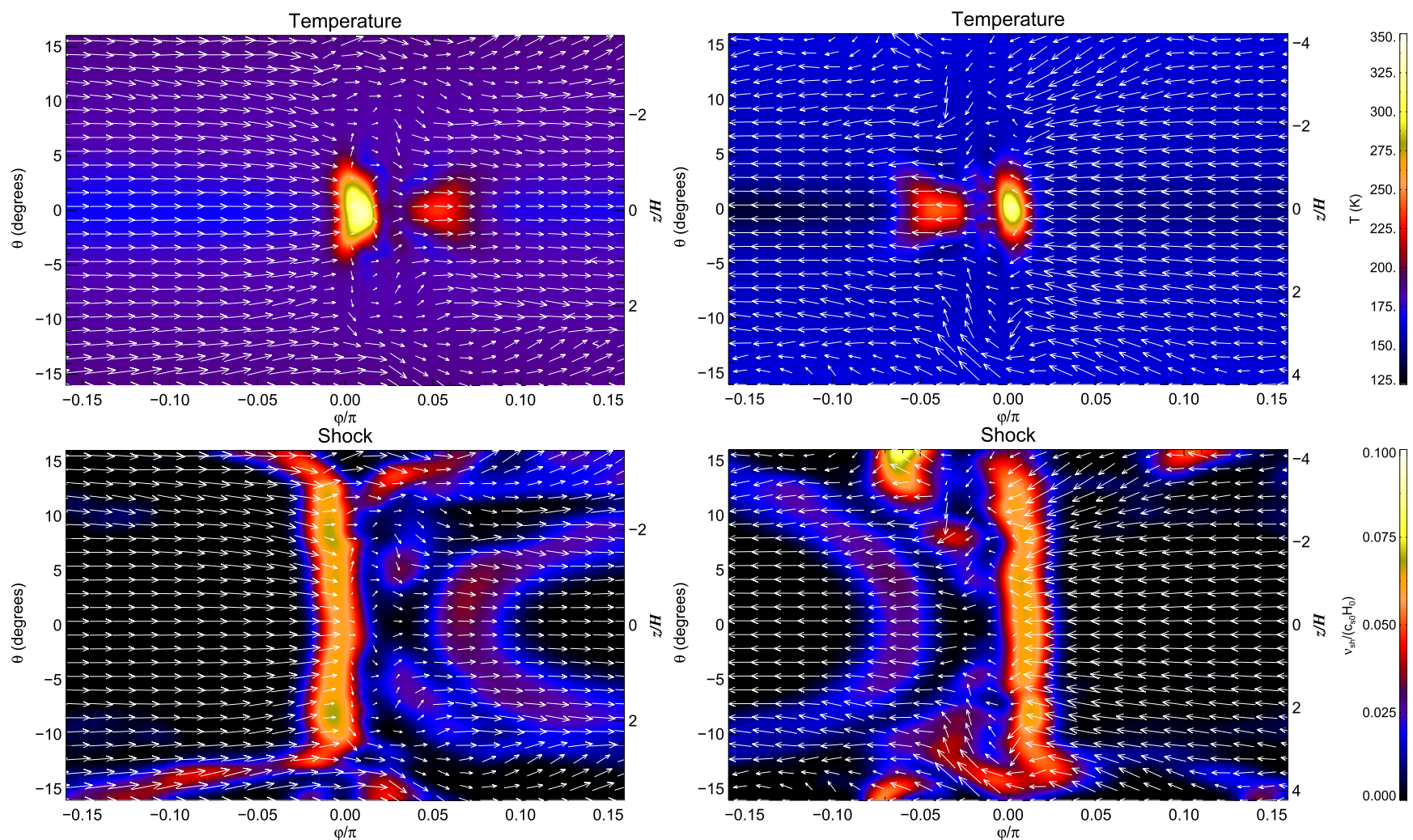

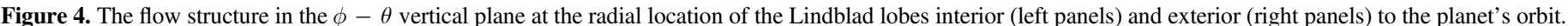

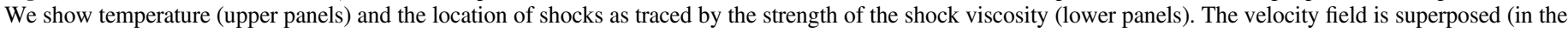

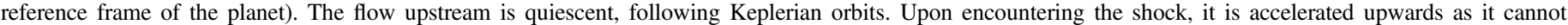

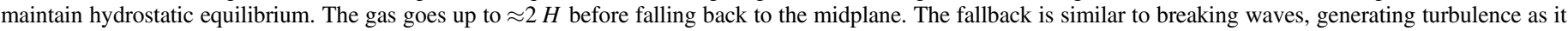

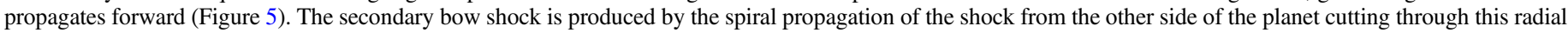
location.

adiabatic gradient is

$$
\left(\frac{d \ln T}{d \ln P}\right)_{\mathrm{ad}}=\frac{\gamma-1}{\gamma}
$$

Gradients steeper than this will generate convection. We plot in the lower panel of Figure 7 the quantity $d \ln T / d \ln P$ along the meridional axis at $r=9 \mathrm{AU}$, which is near the center of the blob. The dotted line traces the value of the adiabatic gradient. We see that the gradient is indeed superadiabatic in the blob. We conclude that we are seeing convection. A planet generates not only turbulence due to breaking waves at its Lindblad lobes, but also heats material that will propagate outward, generating convection far away from its orbit.

Finally, we notice that the upstream and downstream temperatures $(175 \mathrm{~K}$ and $450 \mathrm{~K}$, respectively) straddle the water ice sublimation point. This implies that ice particles passing through the hot shocks will sublimate. The timescale of sublimation can be estimated as $\tau_{\text {subl }} \sim R_{\mathrm{p}} \rho_{\text {。 }} v_{\text {vap }} / P_{\text {sat }}$ (Ros \& Johansen 2013), where $R_{\mathrm{p}}$ and $\rho_{\mathrm{e}}$ are the radius and internal density of the ice particles, respectively, $v_{\text {vap }}$ is the thermal speed of water vapour, and $P_{\text {sat }}$ is the saturation vapour pressure of water (for which we use the expression by Bauer et al. 1997). In the outer shock ( $T \approx 350 \mathrm{~K})$, the sublimation timescale is $\sim 1 \mathrm{~s}$ for a decimeter-sized ice ball, meaning that the evaporation is instantaneous. Yet, the water vapour will quickly recondense downstream. Assuming that the sublimated ice release small silicate grains, condensation would mainly take place on the grains' surfaces (not on larger pebbles and planetesimals), because smaller particles have a larger surfacearea/mass ratio. Therefore, the net effect is the production of a large number of small silicate grains coated by ice in the flow downstream.

This sublimation process can potentially affect the size and spatial distribution of ice particles around the planet. At around $5 \mathrm{AU}$, micron-sized ice grains grow to decimeter-sized balls on a timescale of $\sim 1000$ years (e.g., Brauer et al. 2008; Okuzumi et al. 2012). By contrast, the Lindblad lobes sweep disk gas along their orbits on a much shorter timescale, $\sim 2 \pi r / c_{s} \sim 100$ year. Therefore, ice particles are unable to growth to macroscopic bodies near the orbits of the hot shocks. The destruction of macroscopic ice balls is unavoidable even if we consider the radial inward drift of the particles relative to the gas disk (Adachi et al. 1976; Weidenschilling 1977). At $5 \mathrm{AU}$, the radial drift velocity of decimeter-sized particles is $\sim 1 \mathrm{AU} /\left(10^{3}\right.$ year $)$, and therefore the particles are unable to cross the shock regions (of radial extent $\approx 0.5 \mathrm{AU}$ ) without being swept by the shocks. Since the radial drift slows down as the particle size is decreased, the sublimation of radially drifting ice balls might lead to a pileup of dust at the shocks' orbits.

\section{DISCUSSION AND CONCLUSIONS}

In Paper I we showed that high-mass planets produce radial buoyancy-induced turbulence in their parent disks. The source of energy are the disk wakes generated by the planet at its 

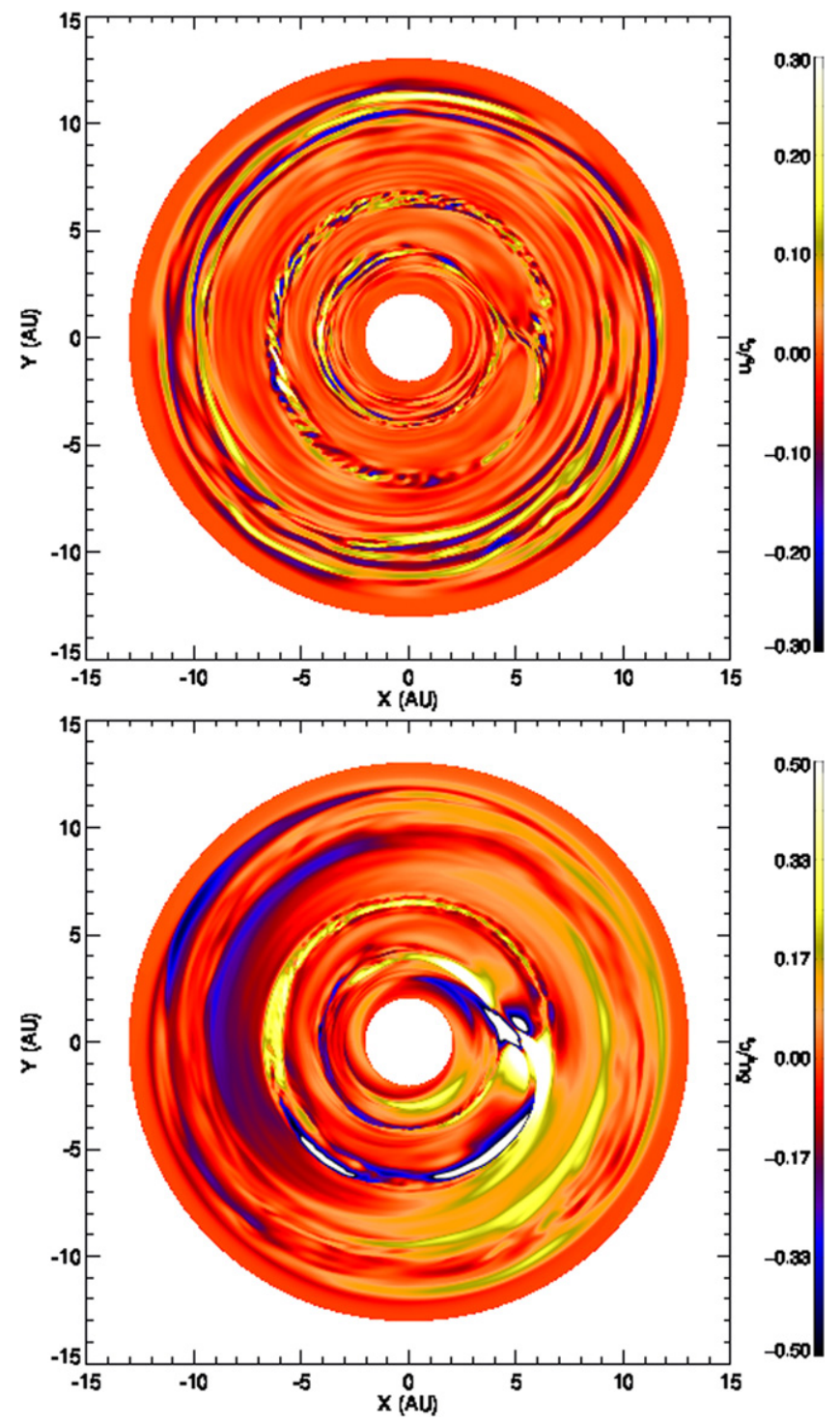

Figure 5. Upper plot: vertical velocity in the midplane. The shocks at the Lindblad lobes generate turbulence along their orbits. The rms velocity of the turbulence is $10 \%$ of the local sound speed, with maximum velocities of $40 \%$ of the sound speed. Lower plot: azimuthal velocity residuals in the midplane. The velocity is supersonic near the planet, with Mach numbers above 2. To highlight the turbulence, we plot it only up to Mach numbers 0.5.

Lindblad resonances, which quickly steepen into shocks for massive planets. So long as the energy is deposited more rapidly than it is dissipated, the temperature rises, leading to instability and turbulence. We found that the turbulence extends throughout most of the disk, and manifests itself more strongly when the cooling is weaker. Indeed, in the adiabatic limit, without cooling, shock dissipation continues to heat the disk until radial buoyancy becomes the dominant motion.

Paper I was a two-dimensional initial study, using Newton (beta) cooling for exploration purposes. In the current paper we describe a 3D study of the same process, with a more sophisticated approximation for treating the radiation field in the disk. Defining a beta cooling as a function of the local optical depth, we reproduce the behavior of adiabaticity $(\beta \gg 1)$ in the midplane, and isothermality $(\beta \ll 1)$ in the atmosphere of a stratified disk.
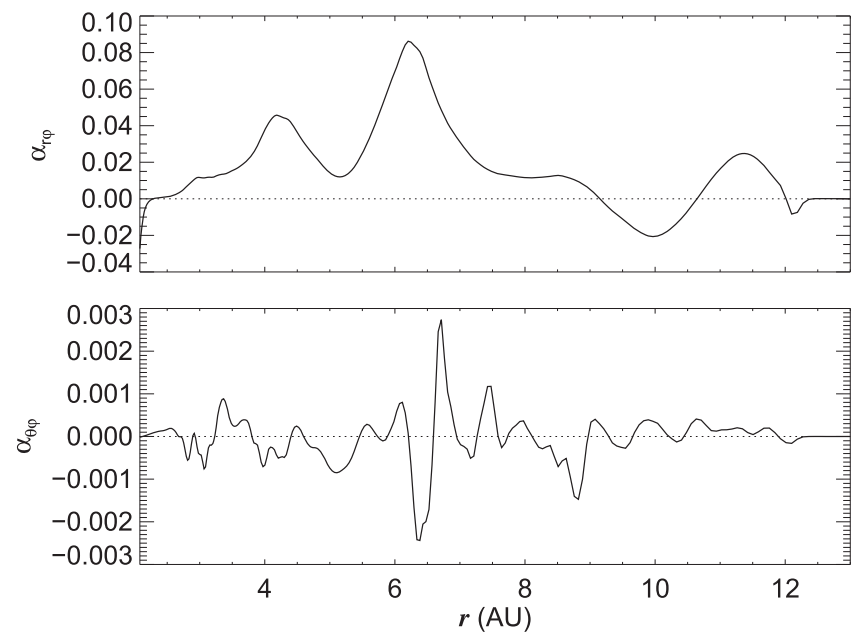

Figure 6. Shakura \& Sunyaev (1973) $\alpha$ viscosity values leading to angular momentum transport in the radial and vertical directions (upper and lower panels, respectively). While the vertical transport is weak, the radial transport of angular momentum reaches an average value of $\approx 0.05$ in the inner Lindblad lobe, and $\approx 0.1$ in the outer one. This should lead to strong accretion, with $v_{\mathrm{rms}} \equiv \sqrt{|\alpha|} c_{s} \approx 0.3 c_{s}$.

In the model presented, the optical depth $\tau$ and the cooling time $\beta$ around the planet's orbit are of the order of 100. For this model, we find that the turbulence continues to occur in $3 \mathrm{D}$, albeit for other reasons than in 2D. From 2D to 3D the main difference is the extra degree of freedom given by the vertical direction, so that as the gas is heated, it expands vertically, which makes the shocks weaker (e.g., Bate et al. 2003). The vertical expansion gives rise to tall shock columns, extending throughout the disk, from the midplane to the atmosphere. These were referred to by Boley \& Durisen (2006) as shock bores. We find that this process occurs at the radii whence the planet spiral wake is launched. The shocks produce hot lobes which, for our parameters, reach about $450 \mathrm{~K}$, three times the temperature of the quiescent gas at the same orbit.

The temperature rise occurs in the midplane, where the disk is more adiabatic, as expected. As the gas meets the shock, it is accelerated upwards. Falling back onto the disk it leads to breaking waves. These waves in turn give rise to turbulence in the full azimuthal extent around the orbital location of each Lindblad lobe. We measure the resulting accretion stress by the Shakura-Sunyaev parameter, finding rapid angular momentum transport with $\alpha$ about 0.05 in the inner lobe, and $\approx 0.1$ in the outer. This means locally $v_{\mathrm{rms}} \approx 0.3 c_{s}$.

We also notice that as the heated gas expands outwards, radially and vertically, it leads to structure in the meridional plane far outward from the planet that appears to be convection. Indeed, we find that these features are associated with localized blobs of high entropy around the midplane in the outer disk. Measuring the entropy gradient, we also find these regions to be superadiabatic, confirming susceptibility to convection. We thus predict that shocks from high-mass planets produce three main features in disks: hot lobes at the locations of the Lindblad resonances, strong turbulence at these radii producing substantial accretion flows, and convection far beyond the radius of the planet.

We compare the maximum temperature of $450 \mathrm{~K}$ we find in the model with that needed to explain the unpolarized bright spiral feature in HD 100546. To determine the latter, we consider the magnitudes in the $H$ (Currie et al. 2015) and $L^{\prime}$ 
$\left(\mathrm{s}-\mathrm{s}_{0}\right) \times 10^{5}$
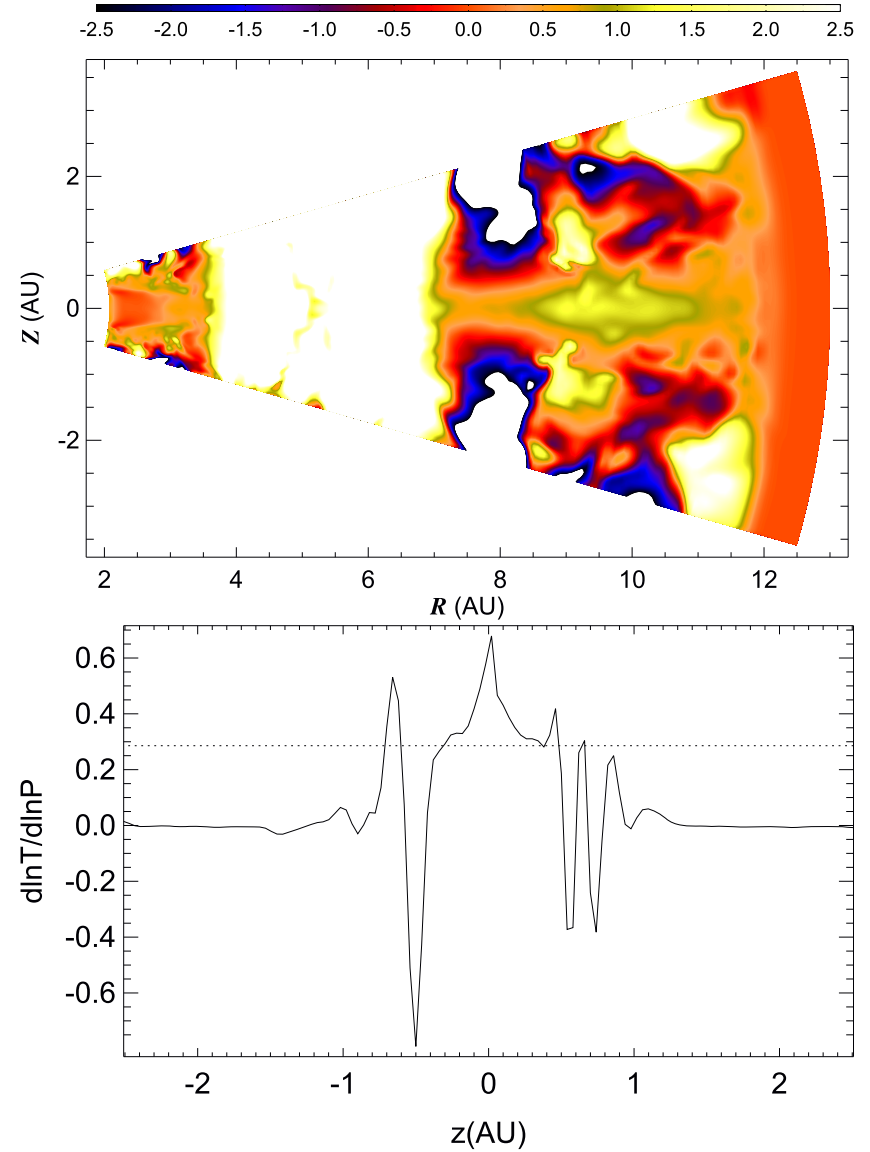

Figure 7. Upper: entropy in the meridional plane that passes through the planet, compared to the initial condition. The plot is saturated, to avoid domination by the structures near the planet. Figure 3 shows structure in the outer disk that appears to be convection. Indeed, a blob of enhanced entropy is seen in the midplane, from about 8.5 to $11 \mathrm{AU}$. Lower: we check the gradient $d \ln T / d \ln P$ (solid line) against the adiabatic gradient (dotted line) at $r=9 \mathrm{AU}$, i.e., cutting through near the center of high entropy blob. The gradient is superadiabatic in the blob, so we conclude that indeed a high-mass planet generates not only turbulence in the Lindblad lobes but also convection far from its orbital radius along the generated spiral.

bands (Currie et al. 2014), and fit blackbody curves to these two data points. To determine the magnitude of the spiral feature we use the quoted magnitudes of HD 100546b in Currie et al. 2014, 2015), and scale the flux according to the signal-tonoise maps (Figure 2 of both works). The magnitudes of HD $100546 \mathrm{~b}$ are $13.06 \pm 0.51$ in the $L^{\prime}$ band, and $19.40 \pm 0.32$ in the $H$ band. For the $L^{\prime}$ band the signal-to-noise is 5 in the planet and 3 in the arm. For the $H$ band, the signal-to-noise is 6 in the planet and 2.5 in the arm. This yields magnitudes of 20.35 and 13.61 for the spiral arm in the $H$ and $L^{\prime}$ bands, respectively. We plot these magnitudes in Figure 8; the data in abscissa are the centers of the $H$ and $L^{\prime}$ bands, 1.65 and $3.5 \mu \mathrm{m}$, respectively. The best fit yields $465 \pm 40 \mathrm{~K}$. The $40 \mathrm{~K}$ standard deviation in temperature is given by the temperatures that encompass the $1 \sigma$ error bars of the magnitudes. The Planckians at 1000, 800 and $600 \mathrm{~K}$ are shown for comparison. The temperature we just estimated is remarkably close to what we find in the model. Yet, we stress that in the model this temperature is confined to

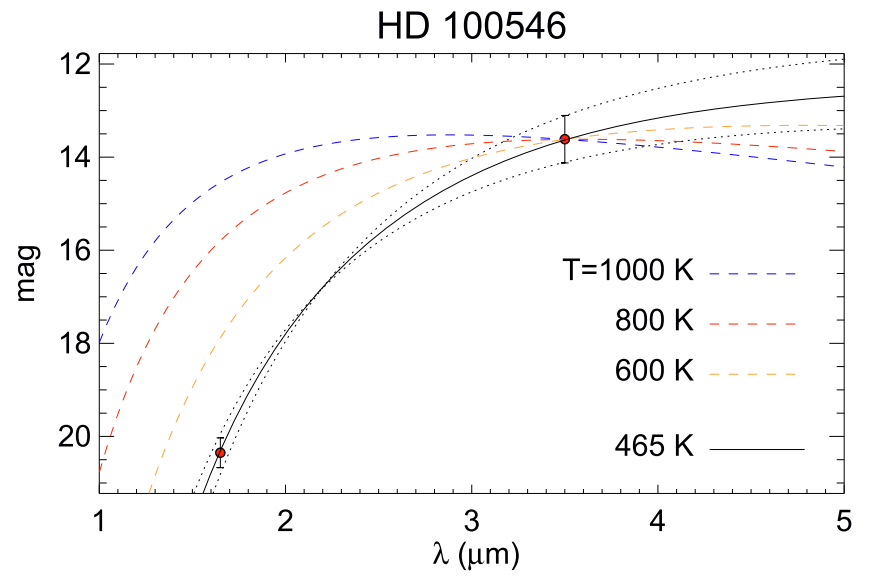

Figure 8. Temperature estimate for the unpolarized bright spiral feature in HD 100546. The data is taken from Currie et al. (2014), and Currie et al. (2015) for the, $L^{\prime}$ and $H$ bands, respectively (see text). The curves shown are blackbody curves. The best fit yields $465 \pm 40 \mathrm{~K}$; the dotted lines mark the $1 \sigma$ standard deviation of $40 \mathrm{~K}$. The Planckians of 1000,800 and $600 \mathrm{~K}$ that pass by the $L^{\prime}$ data point are shown for comparison. The best fit temperature is remarkably close to the maximum temperature of $450 \mathrm{~K}$ we find in our model.

$2 H$ around the midplane, whereas in the HD 100546 observation it occurs in the disk surface.

It is pertinent at this point to explicitly highlight a number of limitations of our model. In our simple approach for the cooling, radiation emitted from the hot lobes simply disappears instead of heating the overlying material. A more complete treatment is worthwhile, to see whether enough shock heat is transferred to the photosphere to be observed. For future work, we intend to do ray-tracing for short-wavelength absorption and flux-limited diffusion for long-wavelength cooling, as in Flock et al. (2013). Another limitation is that we smooth the planet potential at the Hill radius, for numerical stability. This means that the planet potential is much shallower than in reality, so we actually underestimate the magnitude of the shocks and therefore also the temperature enhancement. Reducing the amount of smoothing of the planet's potential would deepen the gravitational well and yield more heating. Finally, in computing the optical depth we take the smaller of the upper and lower values, which makes the midplane cooling too slow by a factor of two. Replacing $\tau_{\text {eff }}$ instead by the inverse of the sum of the inverses of the lower and upper value would give a better estimate. These two corrections are in opposite directions, though, which will tend to minimize their effect. Nevertheless, they should be checked in a future study.

The simulations presented here were carried out using the Stampede cluster of the Texas Advanced Computing Center (TACC) at The University of Texas at Austin through XSEDE grant TG-AST140014. M-MML was partly supported by NASA grant NNX14AJ56G and the Humboldt Foundation. We acknowledge discussions with Thayne Currie and thank the anonymous referee for helpful comments. This work was performed in part at the Jet Propulsion Laboratory, California Institute of Technology. N.J.T. was supported by grant 13OSS13-0114 from the NASA Origins of the Solar System program. 


\section{REFERENCES}

Adachi, I., Hayashi, C., \& Nakazawa, K. 1976, PThPh, 56, 1756

Baruteau, C., Crida, A., Paardekooper, S.-J., et al. 2014, in Protostars and Planets VI, ed. H. Beuther et al. (Tucson: Univ. Arizona Press), 914

Bate, M. R., Lubow, S. H., Ogilvie, G. I., \& Miller, K. A. 2003, MNRAS, 341,213

Bauer, I., Finocchi, F., Duschl, W. J., Gail, H.-P., \& Schloeder, J. P. 1997, A\&A, 317, 273

Bell, K. R., Cassen, P. M., Klahr, H. H., \& Henning, T. 1997, ApJ, 486, 372

Benisty, M., Juhasz, A., Boccaletti, A., et al. 2015, A\&A, 578, 6

Boley, A. C., \& Durisen, R. H. 2006, ApJ, 641, 534

Brauer, F., Dullemond, C. P., \& Henning, T. 2008, A\&A, 480, 859

Bryden, G., Chen, X., Lin, D. N. C., Nelson, R. P., \& Papaloizou, J. C. B. 1999, ApJ, 514, 344

Currie, T., Cloutier, R., Brittain, S., et al. 2015, ApJ, 814, 27

Currie, T., Muto, T., Kudo, T., et al. 2014, ApJ, 796, 30

D’Angelo, G., Henning, T., \& Kley, W. 2003, ApJ, 599, 548

de Val-Borro, M., Edgar, R. G., Artymowicz, P., et al. 2006, MNRAS, 370,529

Dong, R., Hall, C., Rice, K., \& Chiang, E. 2015, ApJ, 812, 32

Flock, M., Fromang, S., González, M., \& Commerçon, B. 2013, A\&A, 560, 43

Garufi, A., Quanz, S. P., Avenhaus, H., et al. 2013, A\&A, 560, 105

Gomez, G. C., \& Cox, D. P. 2004, ApJ, 615, 744

Goodman, J., \& Rafikov, R. R. 2001, ApJ, 552, 793
Haugen, N. E. L., Brandenburg, A., \& Mee, A. J. 2004, MNRAS, 353, 947 Hayashi, C. 1981, PThPS, 70, 35

Horn, B., Lyra, W., Mac Low, M.-M., \& Sándor, Z. 2012, ApJ, 750, 34 Hubeny, I. 1990, ApJ, 351, 632

Isella, A., Natta, A., Wilner, D., Carpenter, J. M., \& Testi, L. 2010, ApJ, 725,1735

Kley, W. 1999, MNRAS, 303, 696

Lubow, S. H., Seibert, M., \& Artymowicz, P. 1999, ApJ, 526, 1001

Lyra, W., Johansen, A., Klahr, H., \& Piskunov, N. 2008, A\&A, 479, 883

Lyra, W., Paardekooper, S.-J, \& Mac Low, M.-M. 2010, ApJ, 715, 68

McNally, C. P., Lyra, W., \& Passy, J.-C. 2012, ApJS, 201, 18

Miki, S. 1982, PThPh, 67, 1053

Muto, T., Grady, C. A., Hashimoto, J., et al. 2012, ApJ, 748, 22

Ogilvie, G. I., \& Lubow, S. H. 2002, MNRAS, 330, 950

Okuzumi, S., Tanaka, H., Kobayashi, H., \& Wada, K. 2012, ApJ, 752, 106

Rafikov, R. R. 2002, ApJ, 569, 997

Richert, A. J. W., Lyra, W., Boley, A. C., Mac Low, M.-M., \& Turner, N. 2015, ApJ, 804, 95

Ros, K., \& Johansen, A. 2013, A\&A, 552, A137

Semenov, D., Wiebe, D., \& Henning, Th. 2004, A\&A, 417, 93

Shakura, N. I., \& Sunyaev, R. A. 1973, A\&A, 24, 337

von Neumann, J., \& Richtmyer, R. D. 1950, JAP, 21, 232

Weidenschilling, S. J. 1977, MNRAS, 180, 57

Zhu, Z., Dong, R., Stone, J. M., \& Rafikov, R. R. 2015, arXiv:1507.03599 